\title{
Can We Make Time for Physical Activity? Simulating Effects of Daily Physical Activity on Mortality
}

\author{
Geoff Rowe, ${ }^{1}$ Mark S. Tremblay, ${ }^{2,3}$ and Douglas G. Manuel ${ }^{1,4,5}$ \\ ${ }^{1}$ Health Analysis Division, Statistics Canada, Ottawa, ON, Canada K1A 0T6 \\ ${ }^{2}$ Healthy Active Living and Obesity Research Group, Children's Hospital of Eastern Ontario Research Institute, \\ Ottawa, ON, Canada K1H $8 L 1$ \\ ${ }^{3}$ Departments of Pediatrics, Human Kinetics, and Epidemiology and Community Medicine, University of Ottawa, \\ Ottawa, ON, Canada K1N 6N5 \\ ${ }^{4}$ Ottawa Hospital Research Institute, University of Ottawa, Ottawa, ON, Canada K1N 6N5 \\ ${ }^{5}$ Departments of Family Medicine and Epidemiology and Community Medicine, University of Ottawa, \\ Ottawa, ON, Canada K1N 6N5
}

Correspondence should be addressed to Geoff Rowe, geoff.rowe@statcan.gc.ca

Received 21 October 2011; Revised 22 March 2012; Accepted 23 April 2012

Academic Editor: Kimberley Edwards

Copyright ( 2012 Geoff Rowe et al. This is an open access article distributed under the Creative Commons Attribution License, which permits unrestricted use, distribution, and reproduction in any medium, provided the original work is properly cited.

Background. The link between physical activity and health outcomes is well established, yet levels of physical activity remain low. This study quantifies effects on mortality of the substitution of low activity episodes by higher activity alternatives using time-use data. Methods. Sample time profiles are representative of the Canadian population $(n=19,597)$. Activity time and mortality are linked using metabolic equivalents(METs). Mortality risk is determined by peak daily METs and hours spent sedentary. The impact of altering activity patterns is assessed using simulated life expectancy. Results. If all leisure screen time was replaced with an equal amount of time spent going for a walk, an increase in life expectancy of about 2.5 years (95\% CI, 1.4 to 3.8) would be expected. No other activity category would have as large as an effect. Conclusions. Reducing leisure screen time has a large effect, because seniors particularly have a large potential for mortality reduction and watch more television than other age groups. The general problem of inactivity cannot be solved simply by reallocating time to more active pursuits, because daily activity patterns can be heterogeneous or fragmented and activities may be nondiscretionary (e.g., work or childcare).

\section{Introduction}

The positive relationship between physical activity and health is well established [1-5], yet levels of physical activity and fitness remain low [6-9], while obesity rates are high, [9, 10] collectively threatening the persistent increase in life expectancy enjoyed over the past century [11]. Physical activity is associated with a decreased risk of mortality, cardiovascular disease, diabetes, colon cancer, osteoporosis, depression, and other chronic disease conditions [1-5], making it an important health promoting behaviour and a priority for intervention. Accumulating evidence shows that sedentary behaviours, independent of physical activity levels, are associated with increased risk of cardiometabolic disease, all-cause mortality, and a variety of physiological and psychological problems [12-17]. Therefore, to maximize health benefits, approaches to resolve the inactivity crisis should attempt to both increase deliberate physical activity and decrease sedentary behaviours.

Physical inactivity is pervasive, persistent, and a challenge to overcome. It is generally believed that a multilevel, multisectoral approach is required to increase population levels of physical activity [18]. Ultimately, resolving the problem of inactivity requires a sustained change in individual daily activity patterns. The feasibility of such changes depends on what competing activities there are and how daily lives are structured. 
The term " "determinant" has been used broadly and imprecisely" [19] in the study of physical activity. A great many factors have been proposed as candidates for a comprehensive theory of the influences on activity patterns: including demographic and biological factors, psychological, cognitive, and emotional factors, behavioural attributes and skills, social and cultural factors, and physical environment factors $[19,20]$. Among these time use $[21,22]$ and characteristics of the built environment $[23,24]$ deserve special consideration, because their potential influence can be specific to a particular point in time and/or location (e.g., the conditions of one person's work may unavoidably limit physical activity for a substantial period of the day; the location of another person's work may not provide opportunities for walking over lunch hour).

Time and location are complementary microfoundations of activity outcomes, but teasing their effects out of data is especially challenging. Unusually detailed data sets may be required, if individual-level heterogeneity is not to be averaged out of existence in the course of analysis. SimObesity [25], a model of obesogenic variables and outcomes, required a spatial microsimulation model to integrate the data necessary to reveal patterns of spatial variation at the neighbourhood level in Leeds, UK. The work reported here exploits a rich set of time use data $[26,27]$ with limited spatial resolution.

The unified modeling of time and location may be most highly developed in urban planning and transportation studies, where "active commuting" is an important topic, in its own right. Urban planners and transportation engineers have begun to recognize the need to incorporate time use strategies in their simulation models of mode choice and traffic flow [28]. For example, replacement of the concept of a trip (one origin and one destination) with that of a tour (one origin and a sequence of destinations) [29] acknowledges that-for example- the commute to and from work may also serve shopping and/or child care needs with obvious implications for active choices. However, a model of all interacting influences on activity would require still greater complexity to deal with effects at the levels of "gene, neurobiology, psychology, family structure and influences, social context and social norms, environment, markets, and public policy" [30].

For the present, with a large sample of quantifiable daily physical activity and sedentary behaviour profiles, and an understanding of the relationships among physical activity, sedentary behaviours, and health outcomes, feasible microsimulation models can be used to explore predicted changes in health outcomes associated with hypothetical population changes in behaviours (e.g., increased walking and/or decreased television viewing). The relative impact of simulated behaviour changes can be explored using this methodology. The time use portion of the General Social Survey (GSS) conducted by Statistics Canada [26, 27] provides information to inform such simulations. Therefore, the purpose of this investigation was to construct a static physical activity simulation model to investigate changes in simulated period life expectancy that could result from reallocations of time spent in various daily activities.

\section{Methods}

Time use diaries are used both to evaluate the change in population health arising from altered activity patterns and to assess the variance of those potential improvementsvariance that ultimately arises from the heterogeneity of time use between individuals, over days of the week, and across seasons of the year. It is the combination of individual level time use data and Monte Carlo simulation methods that makes it possible both to estimate the expected potential change in life expectancy from a specific behavioural change and to estimate the variance of those estimates of change.

2.1. Time Use Data. The 2005 GSS collected data on the daily activities of Canadians living in private dwellings $(n=$ $19,597)$. Information was collected by asking respondents to report their daily activities sequentially during the course of a 24 hour reference day starting at 4:00 am. For each activity respondents were asked the start and end time of the activity, where the activity took place, and who the respondent was with at the time.

In order to facilitate calculation of the variances of survey-based estimates, GSS data files are made available together with sets of bootstrap weights. These weights allow replicate estimates to be calculated. The variance among these replicates accounts for the effect of the complex survey design.

2.2. Imputed Metabolic Equivalents (METs). METs were used to quantify the intensities of activities for each individual daily behaviour profile and so facilitate classification of activities into broad activity levels: sedentary, light, moderate, or vigorous. The mappings of MET values to activity codes were drawn from the "Compendium of Physical Activities" [31], an approach that was modelled after a similar exercise with the American Time Use Survey [32]. In the present study, 158 different activities could be distinguished in terms of METs, with an additional 50 occupational groups providing different MET values for bouts of "Paid Work". (Additional activity categories reflect the location, mode of transport, and social context of activity for each minute of the day.)

2.3. Reporting Errors. The accuracy of self-reported assessments of physical activity is a serious concern. A recent systematic review [33] examined agreement between selfreported (e.g., questionnaire, diary) and directly measured (e.g., accelerometry, doubly labeled water) physical activity in adults, and found low-to-moderate correlations between the two. However, time use diary data, based on a constrained interview process and by identifying concrete activities, has the potential to mitigate these problems in two ways: (1) activity levels may be inferred from known, average characteristics of specific activity types, rather than relying on a subjective general assessment of activity intensity, and (2) the time reference of activity is a specific period of a specific day, rather than a less definite time period (e.g., "activity last month" or "usual activity level"). Nevertheless, METs imputed to these data correspond to averages, and as such do not fully capture individual variability. 


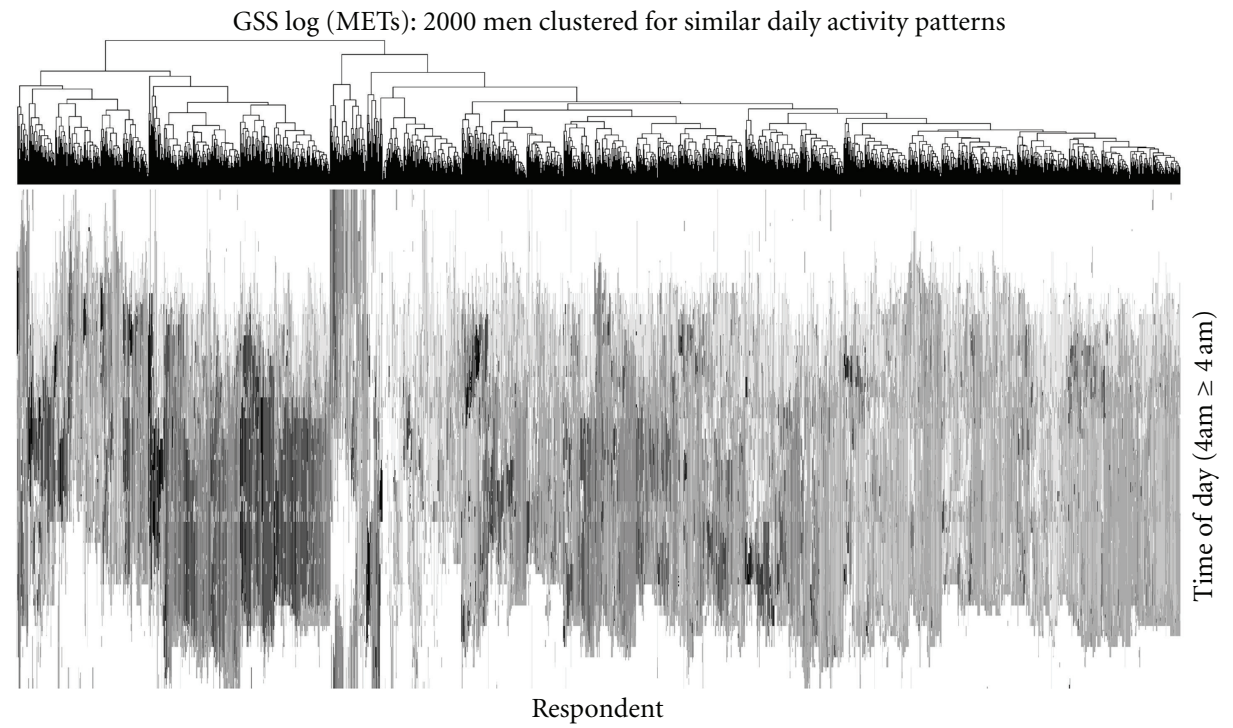

FIGURE 1: Diversity of daily METs. This chart displays time patterns of MET values over a 24-hour period for a random subsample of 2000 person-days reported by male GSS respondents. The vertical dimension represents time of day plotted minute by minute from 4:00 am to 3:59 am (bottom to top), while the horizontal dimension represents individual, sample person-days. The person-days were clustered so that adjacent person-days on the chart were as alike as possible (the tree at the top of the graph displays the linkages among respondent clusters). The shades of grey in the chart code for METs on a log scale with white corresponding to low values (e.g., sleep at the beginning and end of each day) and black corresponding to high values (e.g., vigorous activity at work or in sports). There do not appear to be any compact, widely separated groups, and clustering appears to be determined as much by timing (e.g., shift workers, or those with vigorous activity in the morning and daytime) as it is by intensity.

2.4. Diversity of Daily MET Patterns. Figure 1 clearly illustrates that daily activity as reflected in METs is highly variable both in intensity and in duration. This variability represents the combined effect of interpersonal differences and more systematic differences among days of the week and among the seasons.

2.5. Average Daily Time Use Patterns. Daily life is governed by rhythms which have both metabolic and social underpinnings and that alternate between spells of activity and inactivity of varying duration. Figure 2 illustrates the average organization of daily patterns reflected in GSS data.

The activity intensity scores displayed in Figure 2 reflect the three most important components of deviations from marginal time of day and marginal activity type averages. The scores were determined by Correspondence Analysis [34] applied to a table of GSS activity-time frequencies. The curve labelled "Wake-Sleep" is the most important component as assessed by its "inertia" (an additive contribution to a chi-square statistic measuring deviations from independence of the observed patterns of activity type by time of day). Increasing and positive scores in the "Wake-Sleep" curve tend to be associated with activity type profiles that involve work, school, watching television, and meals. Decreasing and negative scores in the "Wake-Sleep" curve tend to be associated with sleep. The other two curves on the chart have more complex time profiles and different activity type profiles associated with their ups and downs. Together, these three curves represent $82.2 \%$ of total inertia, and, in combination, they demonstrate the fragmentation of time typically found over the course of a day.

The activities most strongly related to the set of three curves include sleep, work, school attendance, meals, watching television, movies, reading, socializing, child care, and commuting. Stable biological factors underlie the regularity of these profiles: factors that operate through sleep/wake cycles, through work/leisure (activity/rest) cycles within the waking phase, and through regularly spaced meal times. Social factors are also at play by means of the institutional constraints imposed by work and through family responsibilities. Sociologists draw all of these together into ordered degrees of time constraint: necessary time, contracted time, committed time, and free time [36]. In relation to the present study, Figure 2 helps draw attention to the degree that such constraints—both biological and social—and the resulting fragmentation of time may limit the potential for behavioural change as it involves daily activity patterns [30].

2.6. Relative Mortality Risks. Relative all-cause mortality risks associated jointly with the intensity of physical activity and with time spent sedentary are used in this study to link activity patterns to mortality outcomes. As is evident from Figure 3, there are appreciable risks associated with being sedentary that are not accounted for by peak activity level.

The primary source [12] of age-sex-adjusted relative risks provided estimates both for time spent sedentary over the course of a typical day and for a dichotomous, self-reported activity status ("active"/“inactive"). The activity dichotomy 

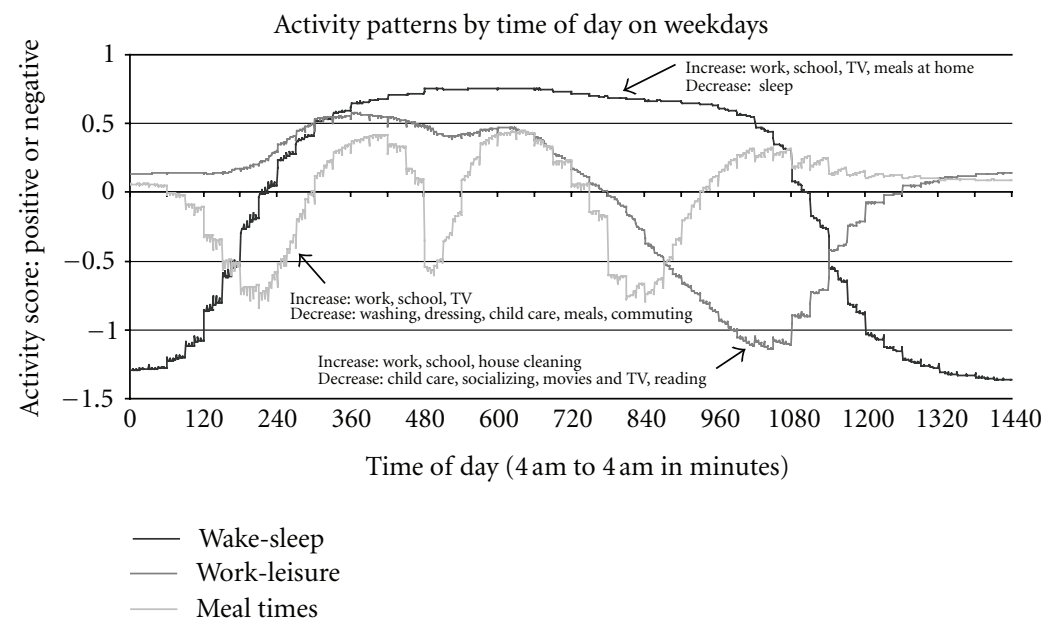

FIGURE 2: Shifting patterns of activity by time of day on weekdays. This chart displays activity intensity scores that vary by time of day and that are associated with particular combinations of activity types. The activity intensity scores were produced using a descriptive dimension reduction technique_correspondence analysis [34]—applied to a table of activity time frequencies representing weekdays. The table comprised 1440 rows - one for each minute of the day-and 182 columns_one for each activity coded in the GSS. Each line on the chart represents a particular time pattern of activity that is associated with a particular profile of activity types.

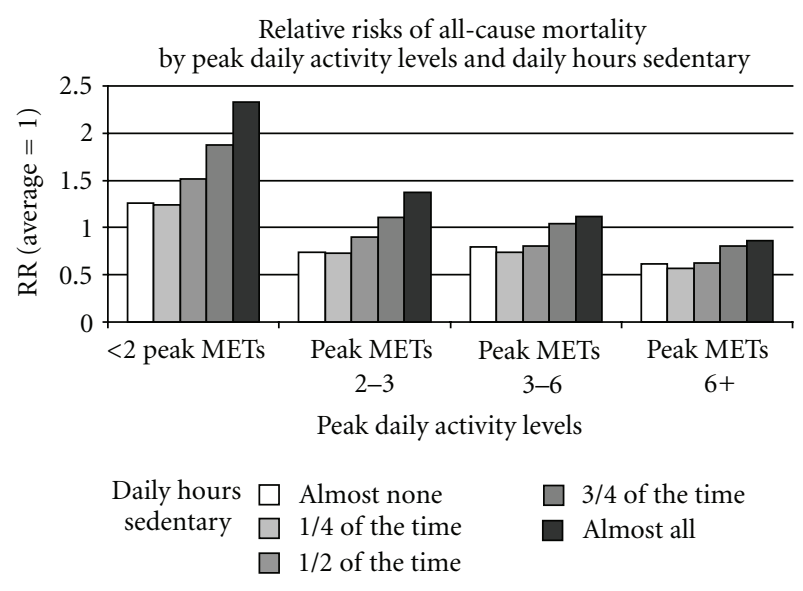

FIGURE 3: Joint relative risks of mortality: peak activities and hours sedentary. For this study, estimates of relative risk were primarily derived from the 1981 Canada Fitness Survey (CFS) augmented with a 12-year mortality followup on 17013 respondents [12]. Secondary estimates, based on a 5-year mortality followup on more than 250,000 participants in the NIH-AARP Diet and Health Study [35], were used to further differentiate between levels of peak activity. These estimates have been rescaled so that the baseline hazard is population average risk, rather than that associated with the lowest activity category.

was extended using data from the secondary source [35] with the assumptions: (1) that the secondary estimates of risk for "vigorous" relative to "moderate" activity levels could be applied to subdivide the primary "active" category, and (2) that the secondary estimates of risk for "light" relative to "sedentary" activity levels could be applied to subdivide the primary "inactive" category.

The summary indicators used to relate relative risks to GSS time profiles were (1) peak activity: the peak MET corresponding to the most intense activity lasting $\geq 30$ minutes and (2) hours sedentary: the total nonsleep hours corresponding to a MET $\leq 2$. Standard MET cut points were used to categorize peak activity as: sedentary, light, moderate, or vigorous. The grouping for hours sedentary was determined so that the percentages in each category corresponded, approximately, to those from the CFS question on time spent sitting [12].

Any discrepancies among activity categories implicitly defined by self-reporting in the two studies from which relative risks were taken or as operationalized using imputed METs will contribute additional bias to the simulations. However, errors in the imputed values of METs will have less of a contribution, because the operationalized activity categories are a coarse classification of METs.

2.7. Baseline Age-Sex-Specific Mortality Hazards. Baseline age-sex-specific mortality hazards are a key parameter in the simulations. They were calculated as occurrence-exposure ratios from Canadian vital statistics data centred on calendar year 2005 [37]. In combination with the relative risks above, these hazards determine the approximate absolute risks associated with physical activity patterns over a complete range of ages. Since these hazards represent averages for the Canadian population, multiplication by appropriate relative risks would produce activity-specific hazards. To obtain appropriate relative risks, the estimates described were rescaled so that the baseline hazard is population average risk, rather than the hazard associated with the lowest activity category.

The impact that the relative risks have on population mortality depends on the prevailing distribution of activity levels. Figure 4 summarises those distributions for the 2005 Canadian population in terms of percentiles of daily average METs and daily peak METs as a function of age. 


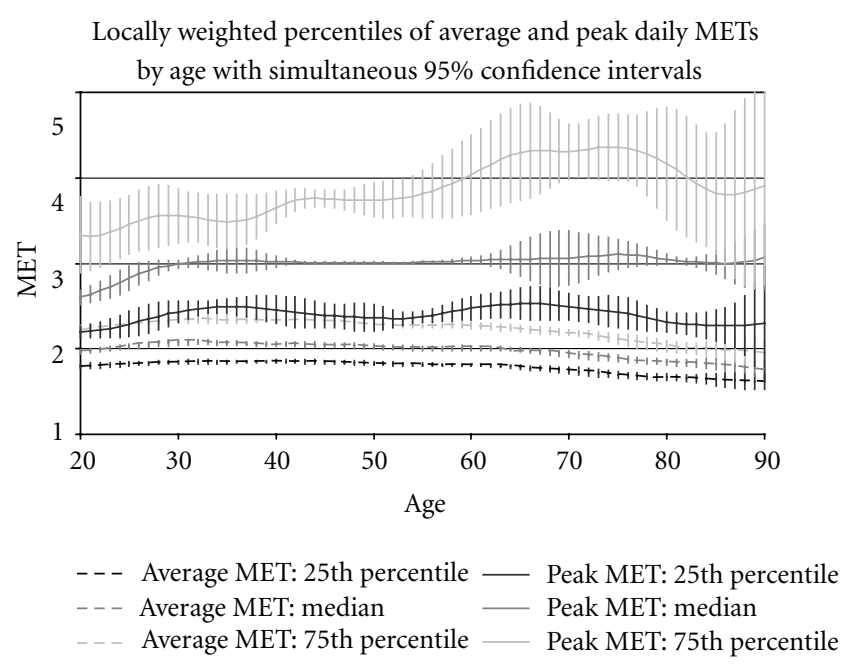

FIgURE 4: Distributions of activity levels in the 2005 Canadian population. These distributional summaries were calculated from average and peak METs of individual GSS respondents using local weights that are a function of age. Average METS represent nonsleep hours only.

The most salient features of these distributional summaries are as follows.

(1) Only about $50 \%$ of the population achieves a peak MET that would put them at or above the moderately active level; however, most of the population is, on average, sedentary over the course of a day.

(2) The most active fraction of the population-as represented by the 75th percentile of peak METsis increasingly active with age (perhaps because of healthy survivors or because retirement can free up hours that, at younger ages, might have been taken up by sedentary work), while declines in average METs with age indicate increased hours spent sedentary among the bulk of seniors.

2.8. Monte Carlo Simulation Design. The Monte Carlo simulations were designed to associate observed or hypothetical patterns of daily activity with implied levels of mortality risk as determined by total person-days distributed among relative risk categories. Simulation of hypothetical deaths within a single year allows both assessment of average agesex specific mortality risks associated with aggregate activity patterns and assessment of the variance in such risks.

A summary of the main steps in each Monte Carlo simulation is as follows (with a more technical description being provided in the appendix).

(1) One GSS respondent at a time is selected as a working case for simulation. (Results reported in this study are based on 10,000,000 such simulated cases for each scenario.) Each working case is randomly selected with replacement and with probability proportional to survey weights.
(2) In order to specify variant scenarios, working time profiles of a sample case are altered by reallocating the time reported for selected activities to alternative activity categories (e.g., from time spent watching television to time spent going for a walk).

(3) Regression equations that relate peak activity and hours sedentary to time profiles are used to adjust the relative risks simulated for each working case in accordance with scenario assumptions.

(4) The mortality hazards on which simulation of death is based are derived using relative risks specific to the appropriate activity categories. In aggregate, these hazards will reflect the hypothetical mortality risks experienced due to the population person-days of activity-specific exposure implied by the scenario.

(5) Simulated estimates of average age-sex-specific hazards are derived from simulated deaths and simulated person-years lived. Period life expectancies at age 15 are derived from those average hazards. (A concern with the validity of applying concepts and methods developed for adults to children motivated the limitation of the simulations to ages 15 and above.)

2.9. Variance Components. An exceptional feature of this simulation model is its capability of assessing the variance of simulated outcomes to decompose that variance into meaningful components. The variance of simulated outputs was accounted for by carrying out sets of 40 separate replicate simulations. Each replicate simulation used a separate bootstrap sample for estimation of regression parameters and for weighting respondent activity profiles. (With 10,000,000 total cases being simulated for each scenario, 40 replicates permits 250,000 cases to be simulated for each replicate.) Variance estimates based on the replicate simulations reflect the combined effect of heterogeneity of time use in the population, sample variability, and errors in estimation of regression coefficients. Carefully specified scenarios (described in the technical appendix) permit the relative contributions of these different sources of variability to be quantified.

\section{Results}

3.1. Life Expectancy for Ages 15+ in the Reference Scenario. The simulated life expectancy estimates at age 15 for Canada in 2005 were $67.9 \pm 0.7$ for women and $63.2 \pm 0.8$ for men. These values agree closely with corresponding official estimates of period life expectancy for men and women at age 15 in 2001 [36]: 67.6 and 62.5 years, respectively.

\subsection{What If Everyone Walked Instead of Their Current Activity}

3.2.1. Scenarios Modifying Time Use Patterns. Figure 5 displays simulation results from a collection of 10 "What If" scenarios that assess the life expectancy differences that would hypothetically result from reallocating all of the time spent in the selected activity categories to going for a walk or a jog 


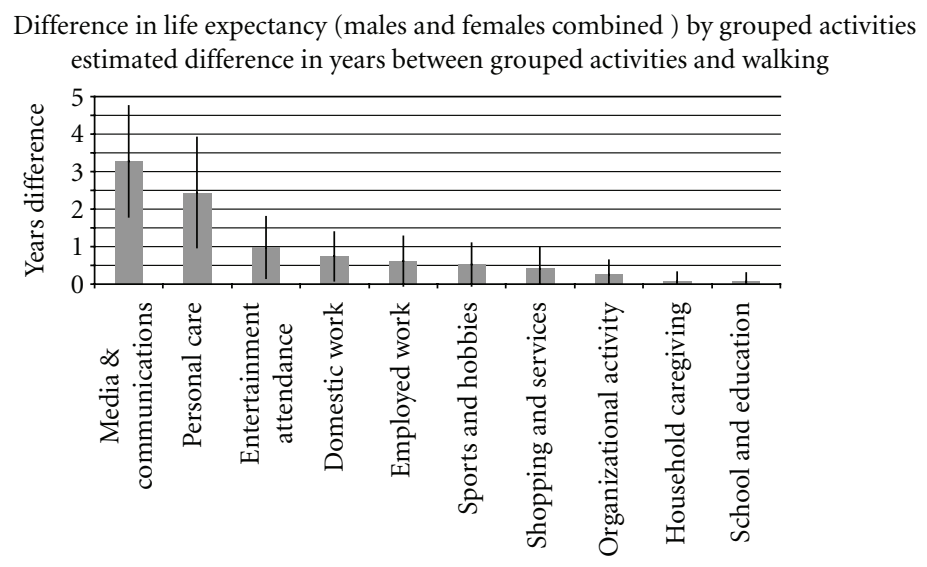

Grouped activity types

FIGURE 5: "What If" scenarios: simulating "going for a walk" instead of their current activity. Current activities comprise 10 grouped activity categories that together account for all nonsleep time in each GSS person-day. The error bars represent $95 \%$ confidence intervals.

(assuming an average of 4.5 METs). These scenarios collectively account for all nonsleep hours over the course of a day. As such, they are intended to provide upper bounds on what might be achieved through time reallocation, rather than as realistic policy objectives.

Only four of the 10 "what if" scenarios result in statistically significant differences in life expectancy and only two appear to stand out in terms of effect size: "Personal Care" and "Media \& Communications." However, each of the latter includes subcategories that would not be appropriate targets for time reallocation. Personal care includes family meals, washing, dressing, and medical care as well as naps and relaxing; media and communications consists of reading, telephone conversations, and letter writing/reading as well as watching television. The only obvious target for a behavioural intervention that is implied by these simulations might be reduction in the sedentary time involving television watching and leisure-time computer use (i.e., leisure screen time). A simulation that reallocated all leisure screen time to going for a walk implied a life expectancy increase of 2.5 years (95\% CI, 1.4 to 3.8 ).

Estimates of differences in life expectancy have relatively large variances. For example, the estimate of the effect of eliminating leisure screen time has a coefficient of variation of nearly $25 \%$. The ultimate source of this variance is the diversity of activity intensity over the course of a day as displayed in Figure 1. This conclusion is reinforced by results of a decomposition of the mean squared error (MSE) of simulation (detailed in the appendix) where the dominant sources of variability were found to be equally divided between sampling variability and the underlying heterogeneity of activity profiles. Variability in parameter estimates-regression coefficients and mortality relative risks - played a minor role.

3.3. Decomposition of the Mean Squared Error. Table 1 assesses the relative contribution of the five main sources of variability as identified by the scenario modifications described in Table 2.
TABLE 1: \% Mean squared error by source: excess total deaths and change in life expectancy.

\begin{tabular}{lcc}
\hline Source & $\begin{array}{c}\text { Excess total } \\
\text { deaths }\end{array}$ & $\begin{array}{c}\text { Change in life } \\
\text { expectancy }\end{array}$ \\
\hline Sampling variability & 48.6 & 53.1 \\
Regression variance & 0.6 & 0.6 \\
Mortality RR variance & 0.1 & 0.1 \\
Rounded reported ages & 0.5 & 4.5 \\
Heterogeneous activity profiles & 50.2 & 41.8 \\
\hline
\end{tabular}

Evidently, the bulk of the empirically based variance in the simulation results can be traced back population heterogeneity or to sampling variability (which is itself a consequence of population heterogeneity). In these simulations, parameter variance (either regression variance or mortality $\mathrm{RR}$ variance) plays a negligible role. Response error-in the form of rounded ages-contributes most to outcomes that are sensitive to the distribution at the oldest ages (i.e., Life expectancy rather than excess deaths).

\section{Discussion}

Together Figures 3 and 4 provide a clear indication that not only is the bulk of the Canadian population exposed to excess mortality risk as a consequence of their patterns of physical activity, but that, for most, the situation worsens with age. This study shows that prevailing patterns of physical activity are associated with a large loss in potential life expectancy. Rather than aiming to have the population vigorously active, important improvements in life expectancy can be gained by replacing current low or sedentary activity with modest exertion. For example, an increase of as much as 2.5 years of life might result if all leisure screen time was replaced by walking. This is an effect size on par with estimates of the elimination of obesity or smoking $[38,39]$. But, it is not 
TABLE 2: Sources of variability identified by a sequence of scenario modifications.

\begin{tabular}{lcl}
\hline Source identified & Reference scenario & Scenario modification \\
\hline Independence* & Reference & $\begin{array}{l}\text { Reallocate time from watching TV to walking } \\
\text { Respondents from bootstrap sample b, while regressions } \\
\text { from }[(\mathrm{b}+1) \text { mod } 40]\end{array}$ \\
Sampling variability* & Watching TV $\geq$ walking & Respondents drawn using full sample weights \\
Regression variance* & Independence & Regressions estimated using full sample weights \\
Combined bootstrap variance & Independence & Combines the two previous scenarios \\
Mortality RR variance & Independence & Set relative risk variance to 0.0 \\
Randomized ages & Combined bootstrap & No perturbation of reported ages \\
Combined randomization & Combined bootstrap & Combines the two previous scenarios \\
Heterogeneous activity profiles & Combined bootstrap & Set all reported activity profiles equal to the mean profile \\
\hline
\end{tabular}

* Required mean bootstrap variance correction.

a realistic policy objective, since it includes much of the Canadian populations' evening leisure time.

An age-by-age examination of simulated improvements in mortality hazards (not shown) indicates that the simulation results arise from age differences in mortality as well as activity patterns. Seniors would benefit more in terms of mortality than other age groups in part because of their higher baseline mortality. But in addition, seniors spend more time sedentary (Figure 4) as also evidenced by higher average hours watching television and so have more scope for improvement in that regard as well. In contrast, the elimination of all vigorous activity (6+ METs) from each time profile has virtually no measurable effect on life expectancy, because vigorous activity is already rare and is concentrated in young (low mortality) ages. So, to achieve the gains promised by the "leisure screen-time" scenario, the behaviour of seniors would have to change more than other age groups.

Thus, reducing the harmful effects of physical inactivity has to involve both changing current daily activity patterns and intensity (increasing physical activity and decreasing sedentary behaviour), while ensuring persistence of those changes throughout the life course. Success in achieving those ends will be demonstrated by an active and healthy population of seniors, since it is at the end of life that the health benefits will be most pronounced.

Opportunities for behaviour intervention differ by age. The feasibility of specific interventions is influenced by how daily lives are structured, while that structure, in turn, is influenced by institutional constraints related to work or school and by constraints related to mutual organization of time among family and friends. These influences may vary over the life cycle.

Patterns of daily time use are highly variable. This variability is directly observable in these data (Figure 1) and could pose problems for effective public health policy implementation. The decomposition of mean squared simulation error implies that about $50 \%$ of the variance in simulated intervention effects may be attributed to population heterogeneity directly. In other words, the confidence intervals on these intervention effects would likely only be $30 \%$ narrower, if the estimates could be based on a census rather than a sample. In that circumstance, substituting time spent walking for most of the broad activity categories shown in Figure 5 would still indicate no significant improvement in mortality. Thus, with this much variability in the population, there is a risk that the impact of interventions that are too narrowly focused or too limited in scope might be dissipated. In fact, achievement of population physical activity objectives may not be feasible through reallocation of time alone. Rather, it may be necessary to find ways to increase the METs associated with common activities, reversing a long-standing trend by investing in technology that is less labour saving rather than more.

Essentially, this study takes relative risks estimated from published prospective mortality studies $[12,35]$ and reexpresses them in terms of life expectancies. However, the special contribution of the study derives from the direct links that are established among mortality risks, intensity of physical activity, and time use patterns. The microdata foundations of these links and the use of Monte Carlo microsimulation techniques permit an evaluation of the variance of hypothesized effects, as well as offering insights into the influence that time use constraints and the diversity of time use might have on the potential success of behavioural interventions aimed at reducing physical inactivity.

But, this study has important limitations. It depends on published estimates of relative risks taken from only two studies [12,35]. Incorporation of estimates from other studies would lead to more robust conclusions. However, despite there being numerous studies testing for an effect of leisure-time physical activity on mortality, there are fewer studies that test effects of more broadly defined activities (including nonleisure time) and fewer still that consider joint effects of activity and time spent sedentary. Another limitation involves the fact that the risk estimates used were adjusted for age and sex only. As such, they can only provide upper bounds on the effect of physical activity on mortality. Adjusting for potential confounding variables would provide better measures of the direct causal effect of physical activity on mortality risk. Even so, the main source [12] of relative risk estimates used in this study indicated that multivariate 
adjustment including smoking and alcohol consumption did not result in any marked change in the relative risks to time spent sitting. Finally, in this study METs are imputed rather than measured and, as such, some of the underlying variability in activity patterns remains unobserved.

In summary, this study demonstrates the value of a novel simulation method that directly relates detailed activity patterns to expected mortality outcomes and their variance, in a manner that enhances the value of the data for health policy purposes.

\section{Appendix}

\section{Technical Specification of the Simulation Model}

This appendix provides a rigorous description of the model by means of an algebraic representation in terms of parameters and outcome measures.

Elements of the model: simulation equations and parameters:

$$
\left.\begin{array}{c}
h_{i(j(b))}^{S}\left(a_{i(j(b))}^{*}, g_{j}\right) \\
=h_{0}\left(a_{i(j(b))}^{*}, g_{j}\right) \exp \left(I\left[P_{i(j(b))}^{S}, H_{i(j(b))}^{S}\right] \theta\right. \\
\left.\quad+\sigma Z_{i(j(b))}-\frac{\sigma^{2}}{2}\right),
\end{array}\right\}
$$

where $b$ is bootstrap replicate sample, $j$ is survey respondent, $i$ is replicate sequence number (number of times sampled), $i(j(b))$ is $i$ th simulation of respondent $j$ within bootstrap sample $b, S$ is scenario identifier, $a$ and $a^{*}$ are observed and randomly perturbed ages, $g$ is gender $(0=$ Female, $1=$ Male $)$, $U^{\prime}$ and $U^{\prime \prime}$ are independent uniform $[0,1]$ pseudorandom numbers, $Z$ is standard normal pseudorandom number, $h_{0}\left(a^{*}, g\right)$ is baseline hazard specific to age $a^{*}$ and gender $g, h_{i(j(b))}^{S}\left(a^{*}, g\right)$ is scenario specific, activity adjusted hazard, $P_{i(j(b))}^{S}$ is scenario specific peak activity, $H_{i(j(b))}^{S}$ is scenario specific hours sedentary, $I[]$ is indicator $(0$ or 1$)$ vector given $P_{j}$ and $H_{j}$ and given cut-points $C^{P}$ and $C^{H}, \theta$ is vector of (log) relative risks, $\sigma^{2}$ is approximate (scalar) variance of (log) relative risks, $X_{j}$ and $X_{j}^{S}$ are observed and scenario specific activity profile vectors, $\beta_{b, a^{*}}^{H}$ is hours sedentary regression coefficients (bootstrap $b$, age $a^{*}$ ), $\beta_{b, a^{*}}^{P}$ is peak activity regression coefficients (bootstrap $b$, age $a^{*}$ ).

Simulation outcomes: deaths and life expectancy at age 15+ deaths:

$$
\begin{aligned}
D_{i(j(b))}^{S} & = \begin{cases}1, & \text { if }\left\langle-\ln \frac{\left(U_{i(j(b))}\right)}{h_{i(j(b)}^{S}}\right\rangle<1 \\
0, & \text { otherwise, }\end{cases} \\
D_{+, b}^{S} & =\sum_{i, j} D_{i(j(b)),}^{S}
\end{aligned}
$$

where $U_{i(j(b))}$ is a uniform $[0,1]$ pseudo-random number, $D_{i(j(b))}^{S}$ is the $i$ th replicate mortality outcome simulated for respondent $j$ in bootstrap sample $b$ under scenario $S$, and $D_{+, b}^{S}$ is the total number of deaths simulated using bootstrap sample $b$ under scenario $S$.

Life expectancy 15+:

$$
\begin{gathered}
\hat{e}_{g}^{S} \approx \sum_{a=15} \frac{\left(\hat{l}_{a+1, g}^{S}+\hat{l}_{a, g}^{S}\right)}{2}, \quad \hat{l}_{a, g}^{S}=\exp \left(-\sum_{x=15}^{a} \hat{h}_{x, g}^{S}\right) \\
\hat{h}_{a, g}^{S}=\frac{\sum_{\substack{a=a_{i(j(b))}^{*} \\
g=g_{j}}} D_{i(j(b))}^{S}}{\sum_{\substack{a=a_{i(j(b))}^{*} \\
g=g_{j}}}^{S} F_{i(j(b))}^{S}}
\end{gathered}
$$

where $F_{i(j(b))}^{S}$ is the fraction of the year lived in the simulation (i.e., 1 if no death was simulated and a fraction otherwise), $\hat{h}_{a, g}^{S}$ is the simulated empirical hazard at age a and for gender $g, \widehat{l}_{a, g}^{S}$ is the simulated survival probability from age 15 to age a for gender $g$, and $\hat{e}_{g}^{S}$ is the simulated life expectancy at age 15 for gender $g$.

The model allows an assessment of outcome variance components that can be ascribed to model parameters. 
Mean squared error (MSE) of simulated excess total deaths:

$$
\begin{gathered}
\operatorname{MSE}\left(S^{\prime}, S\right)=\left(\bar{D}_{+}^{S^{\prime}}-\bar{D}_{+}^{S}\right)^{2}+\hat{\operatorname{Var}}\left(D_{+}^{S^{\prime}}-D_{+}^{S}\right), \\
\bar{D}_{+}^{S^{\prime}}=\frac{\sum_{b=1}^{B} D_{+, b}^{S^{\prime}}}{B}, \quad \bar{D}_{+}^{S}=\frac{\sum_{b=1}^{B} D_{+, b}^{S}}{B}, \\
\operatorname{Var}\left(D_{+}^{S^{\prime}}-D_{+}^{S}\right)= \\
\operatorname{VC}_{S}\left[\frac{\sum_{b=1}^{B}\left(D_{+, b}^{S^{\prime}}-D_{+, b}^{S}\right)^{2}}{B}-\left(\bar{D}_{+}^{S^{\prime}}-\bar{D}_{+}^{S}\right)^{2}\right], \\
\mathrm{VC}_{S}= \begin{cases}25, & \text { if scenario } S \text { requires Mean Bootstrap } \\
1, & \text { otherwise. }\end{cases}
\end{gathered}
$$

A similar, but more involved, calculation determines the MSE of simulated life expectancies.

Variance Components. There are four main sources of variability in the model, apart from that linking a simulated mortality hazard to a simulated mortality outcome:

(1) $i(j(b))$ : sampling variability as reflected in bootstrap replicates,

(2) $\operatorname{Var}\left(\beta_{a}^{H}\right)$ and $\operatorname{Var}\left(\beta_{a}^{P}\right)$ : variance of regression coefficients among bootstrap replicates,

(3) $\sigma^{2}$ : variance of $(\log )$ relative mortality risks, and

(4) $a^{*}$ : randomly perturbing ages fills in gaps and smooths the sample age distribution (which exhibited effects of rounding).

These sources of variability are incorporated into the simulation by

(1) sampling respondents with probability proportional to bootstrap weights,

(2) using regressions estimated from separate bootstrap replicate samples,

(3) explicitly perturbing the relative risk parameter reflecting errors in estimation,

(4) explicitly perturbing the observed age to counter effects of rounding.

A fifth, more fundamental source of variability is the heterogeneity within the population as represented in sampled person-day activity profiles.

The relative importance of source of variability can be assessed by comparing a given scenario $S$ to a modified form of that scenario $S^{\prime}$ involving only one change to the simulation parameters. This is analogous to the determination of the reduction in residual sums of squares attributable to a given variable in a regression equation by deleting one variable at a time and noting the resulting increase in residual sums of squares. More formally, the variance of the difference between statistics generated by the modified and unmodified scenarios can be expressed as

$$
\operatorname{Var}\left(S-S^{\prime}\right)=\operatorname{Var}(S)+\operatorname{Var}\left(S^{\prime}\right)-2 \operatorname{Cov}\left(S, S^{\prime}\right) .
$$

But, if $S^{\prime}$ and $S$ differ because $S^{\prime}$ has one source of variability eliminated (and given that all sources are uncorrelated with each other), then $\operatorname{Cov}\left(S, S^{\prime}\right)=\operatorname{Var}\left(S^{\prime}\right)$; then,

$$
\operatorname{Var}\left(S-S^{\prime}\right)=\operatorname{Var}(S)-\operatorname{Var}\left(S^{\prime}\right)
$$

a direct measure of the variance due to the source of variability that was removed.

However, there are complications: sampling variability and regression variance are correlated, because they are based on matched bootstrap samples; variance estimates associated with bootstrapped sources of variability need correction for the use of a mean bootstrap algorithm, but other sources do not. (To produce each set of mean bootstrap weights, sets of 25 independent, conventional bootstrap weights were average, so, bootstrapped nominal variances had to be multiplied by 25 to produce consistent variance estimates.) Thus, the decomposition of simulation MSE requires a sequence of steps in which appropriate reference scenarios are identified against which to compare scenarios from which specific sources of variability have been removed.

\section{Acknowledgments}

Computer implementation of these simulations was considerably simplified using Statistics Canada's Modgen technology [40]. Thanks are due to Michael Wolfson and other reviewers who provided valuable comments.

\section{References}

[1] United States Department of Health and Human Services, "Physical activity and health: a report of the surgeon general," Tech. Rep., United States Department of Health and Human Services, Centers for Disease Control and Prevention, National Center for Chronic Disease Prevention and Health Promotion, Atlanta, Ga, USA, 1996.

[2] Physical Activity Guidelines Advisory Committee, "Physical activity guidelines advisory committee report," Tech. Rep., United States Department of Health and Human Services, Washington, DC, USA, 2008.

[3] I. Janssen and A. G. LeBlanc, "Systematic review of the health benefits of physical activity and fitness in school-aged children and youth," International Journal of Behavioral Nutrition and Physical Activity, vol. 7, article 40, 2010.

[4] D. E. R. Warburton, S. Charlesworth, A. Ivey, L. Nettlefold, and S. S. D. Bredin, "A systematic review of the evidence for Canada's Physical Activity Guidelines for Adults," International Journal of Behavioral Nutrition and Physical Activity, vol. 7, article 39, 2010.

[5] D. H. Paterson and D. E. R. Warburton, "Physical activity and functional limitations in older adults: a systematic review related to Canada's Physical Activity Guidelines," International Journal of Behavioral Nutrition and Physical Activity, vol. 7, article no. 38, 2010.

[6] R. P. Troiano, D. Berrigan, K. W. Dodd, L. C. Mâsse, T. Tilert, and M. Mcdowell, "Physical activity in the United States 
measured by accelerometer," Medicine and Science in Sports and Exercise, vol. 40, no. 1, pp. 181-188, 2008.

[7] P. T. Katzmarzyk and M. S. Tremblay, "Limitations of Canada's physical activity data: implications for monitoring trends," Applied Physiology, Nutrition and Metabolism, vol. 32, pp. S185-S194, 2007.

[8] M. S. Tremblay, M. Shields, M. Laviolette, C. L. Craig, I. Janssen, and S. C. Gorber, "Fitness of Canadian children and youth: results from the 2007-2009 Canadian Health Measures Survey," Health Reports, vol. 21, no. 1, pp. 7-20, 2010.

[9] M. Shields, M. S. Tremblay, M. Laviolette, C. L. Craig, I. Janssen, and S. C. Gorber, "Fitness of Canadian adults: results from the 2007-2009 Canadian Health Measures Survey," Health Reports, vol. 21, no. 1, pp. 21-35, 2010.

[10] K. M. Flegal, M. D. Carroll, C. L. Ogden, and L. R. Curtin, "Prevalence and trends in obesity among US adults, 19992008," The Journal of the American Medical Association, vol. 303, no. 3, pp. 235-241, 2010.

[11] S. J. Olshansky, D. J. Passaro, R. C. Hershow et al., "A potential decline in life expectancy in the United States in the 21st century," The New England Journal of Medicine, vol. 352, no. 11, pp. 1138-1145, 2005.

[12] P. T. Katzmarzyk, T. S. Church, C. L. Craig, and C. Bouchard, "Sitting time and mortality from all causes, cardiovascular disease, and cancer," Medicine and Science in Sports and Exercise, vol. 41, no. 5, pp. 998-1005, 2009.

[13] N. Owen, A. Bauman, and W. Brown, "Too much sitting: a novel and important predictor of chronic disease risk?" British Journal of Sports Medicine, vol. 43, no. 2, pp. 81-83, 2009.

[14] M. S. Tremblay, R. C. Colley, T. J. Saunders, G. N. Healy, and N. Owen, "Physiological and health implications of a sedentary lifestyle," Physiology, Nutrition and Metabolism, vol. 35, no. 6, pp. 725-740, 2010.

[15] A. V. Patel, L. Bernstein, A. Deka et al., "Leisure time spent sitting in relation to total mortality in a prospective cohort of US adults," American Journal of Epidemiology, vol. 172, no. 4, pp. 419-429, 2010.

[16] D. W. Dunstan, E. L. M. Barr, J. E. Shaw et al., "Response to letters regarding article, 'television viewing time and mortality: the Australian diabetes, obesity and lifestyle study (AusDiab)"," Circulation, vol. 122, no. 13, p. e472, 2010.

[17] M. Inoue, H. Iso, S. Yamamoto et al., "Daily total activity level and premature death in men and women: results from a largescale population-based cohort study in Japan (JPHC Study)," Annals of Epidemiology, vol. 18, no. 7, pp. 522-530, 2008.

[18] "National Physical Activity Plan," http://www.physicalactivityplan.org/, 2010.

[19] A. E. Bauman, J. F. Sallis, D. A. Dzewaltowski, and N. Owen, "Toward a better understanding of the influences on physical activity: the role of determinants, correlates, causal variables, mediators, moderators, and confounders," American Journal of Preventive Medicine, vol. 23, no. 2, supplement 1, pp. 5-14, 2002.

[20] C. Bouchard and P. T. Katzmarzyk, Eds., Physical Activity and Obesity, Pennington Biomedical Research Centre, Baton Rouge, La, USA, 2010.

[21] M. John and S. A. Robert, "No time to lose? Time constraints and physical activity," Working Paper 14513, National Bureau of Economic Research, 2008.

[22] K. Y. Wolin, G. G. Bennett, L. H. McNeill, G. Sorensen, and K. M. Emmons, "Low discretionary time as a barrier to physical activity and intervention uptake," American Journal of Health Behavior, vol. 32, no. 6, pp. 563-569, 2008.

[23] Transportation Research Board, "Does the built environment influence physical activity? Examining the evidence," TRB
Special Report 282, Committee on Physical Activity, Health, Transportation, and Land Use, Transportation Research Board and Institute of Medicine of the National Academies of Science, 2005.

[24] A. A. Lake, T. G. Townshend, and S. Alvanides, Eds., Obesegenic Environments: Complexities, Perceptions and Objective Measures, Wiley-Blackwell, Oxford, UK, 2010.

[25] K. L. Edwards and G. P. Clarke, "The design and validation of a spatial microsimulation model of obesogenic environments for children in Leeds, UK: SimObesity," Social Science and Medicine, vol. 69, no. 7, pp. 1127-1134, 2009.

[26] Statistics Canada, "Overview of the time use of Canadians," General Social Survey on Time Use 12F0080XIE, 2005.

[27] Statistics Canada, "General Social Survey-Time Use, Detailed information for 2005 (Cycle 19) and Data Files," http://www .statcan.gc.ca/cgibin/imdb/p2SV.pl?Function=getSurvey\& SurvId $=4503 \&$ SurvVer $=2 \&$ InstaId $=16848 \&$ InstaVer $=4 \&$ SDDS $=4503 \&$ lang $=e n \& d b=I M D B \& a d m=8 \& d i s=2$.

[28] B. Lenz and C. Nobis, "The changing allocation of activities in space and time by the use of ICT- "Fragmentation" as a new concept and empirical results," Transportation Research A, vol. 41, no. 2, pp. 190-204, 2007.

[29] C. H. Wen and F. S. Koppelman, "A conceptual and methdological framework for the generation of activity-travel patterns," Transportation, vol. 27, no. 1, pp. 5-23, 2000.

[30] D. S. Hamermesh and S. Donald, "The time and timing costs of market work," Working Paper number 13127, National Bureau of Economic Research, 2007.

[31] B. E. Ainsworth, W. L. Haskell, M. C. Whitt et al., "Compendium of physical activities: an update of activity codes and MET intensities," Medicine and Science in Sports and Exercise, vol. 32, no. 9, pp. S498-S504, 2000.

[32] C. Tudor-Locke, T. L. Washington, B. E. Ainsworth, and R. P. Troiano, "Linking the American Time Use Survey (ATUS) and the compendium of physical activities: methods and rationale," Journal of Physical Activity and Health, vol. 6, no. 3, pp. 347-353, 2009.

[33] S. A. Prince, K. B. Adamo, M. E. Hamel, J. Hardt, S. Connor Gorber, and M. Tremblay, "A comparison of direct versus self-report measures for assessing physical activity in adults: a systematic review," International Journal of Behavioral Nutrition and Physical Activity, vol. 5, article no. 55, 2008.

[34] M. J. Greenacre, Theory and Applications of Correspondence Analysis, Academic Press, London, UK, 1984.

[35] M. F. Leitzmann, Y. Park, A. Blair et al., "Physical activity recommendations and decreased risk of mortality," Archives of Internal Medicine, vol. 167, no. 22, pp. 2453-2460, 2007.

[36] "Statistics Canada: Life Tables, Canada, Provinces and Territories: 2000 to 2002," http://www5.statcan.gc.ca/access_acces/ archive.action?loc=/pub/84-537-x/2006001/4227757-eng.pdf.

[37] "Statistics Canada: Canadian vital statistics data," http://www5 .statcan.gc.ca/cansim/directory-repertoire?lang=eng\&groupid= 051.

[38] D. Ås, "Studies of time-use: problems and prospects," Acta Sociologica, vol. 21, no. 2, pp. 125-141, 1978.

[39] S. T. Stewart, D. M. Cutler, and A. B. Rosen, "Forecasting the effects of obesity and smoking on U.S. life expectancy," The New England Journal of Medicine, vol. 361, no. 23, pp. 22522260, 2009.

[40] "Statistics Canada: Microsimulation," http://www.statcan.gc .ca/microsimulation/index-eng.htm. 


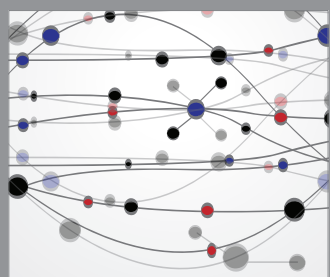

The Scientific World Journal
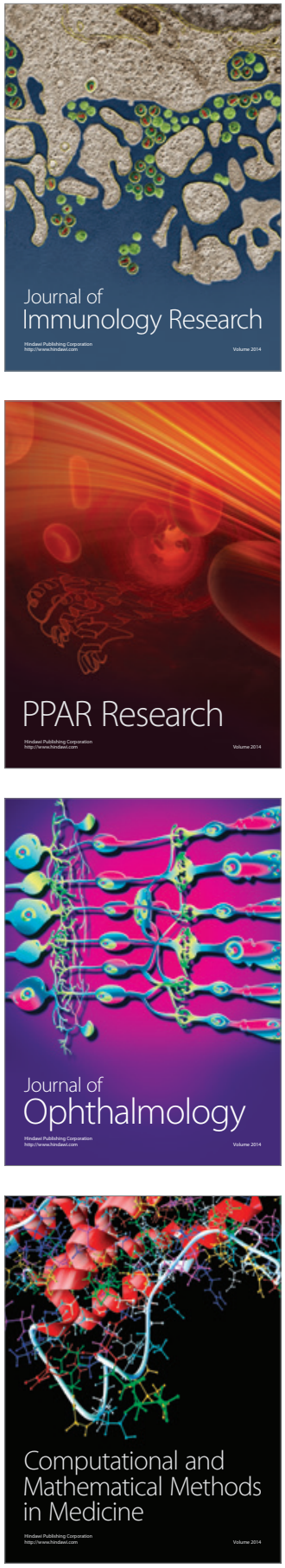

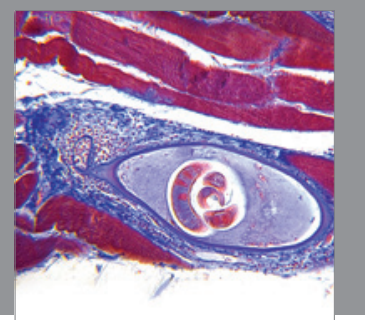

Gastroenterology

Research and Practice
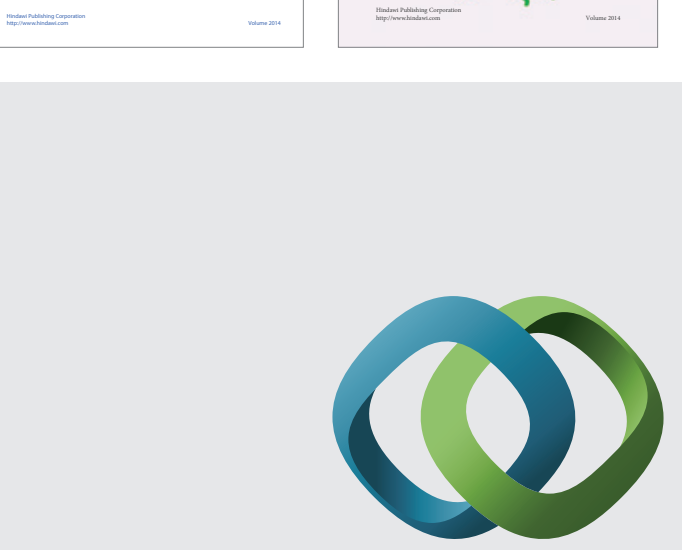

\section{Hindawi}

Submit your manuscripts at

http://www.hindawi.com
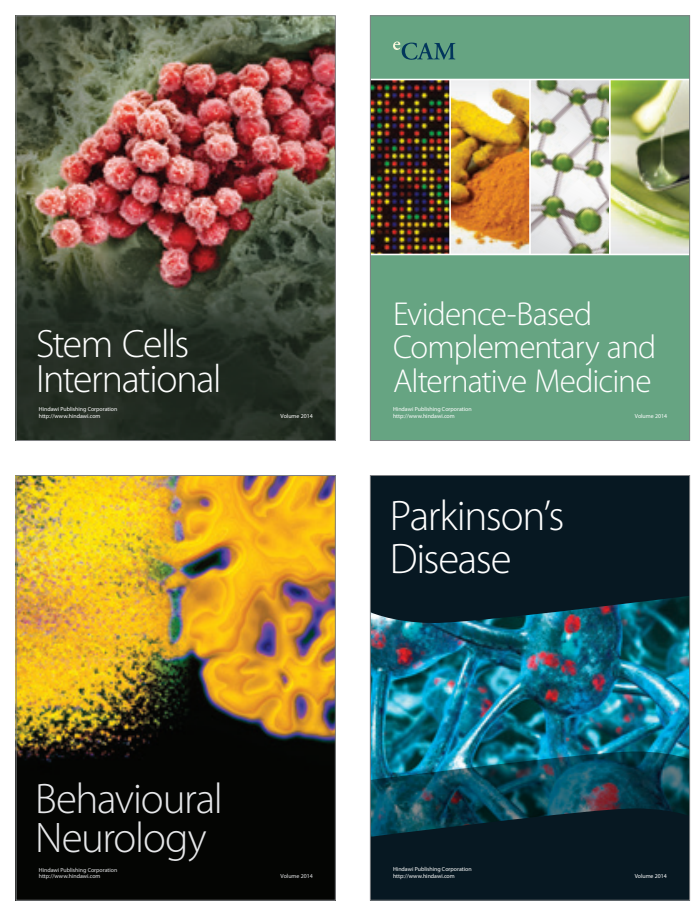

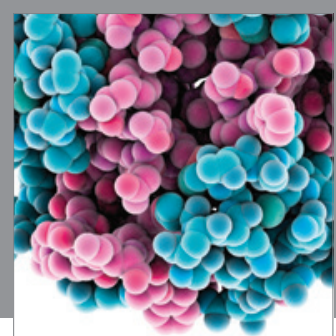

Journal of
Diabetes Research

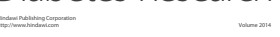

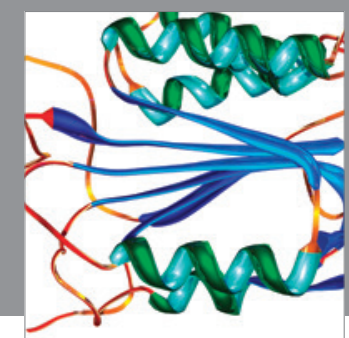

Disease Markers
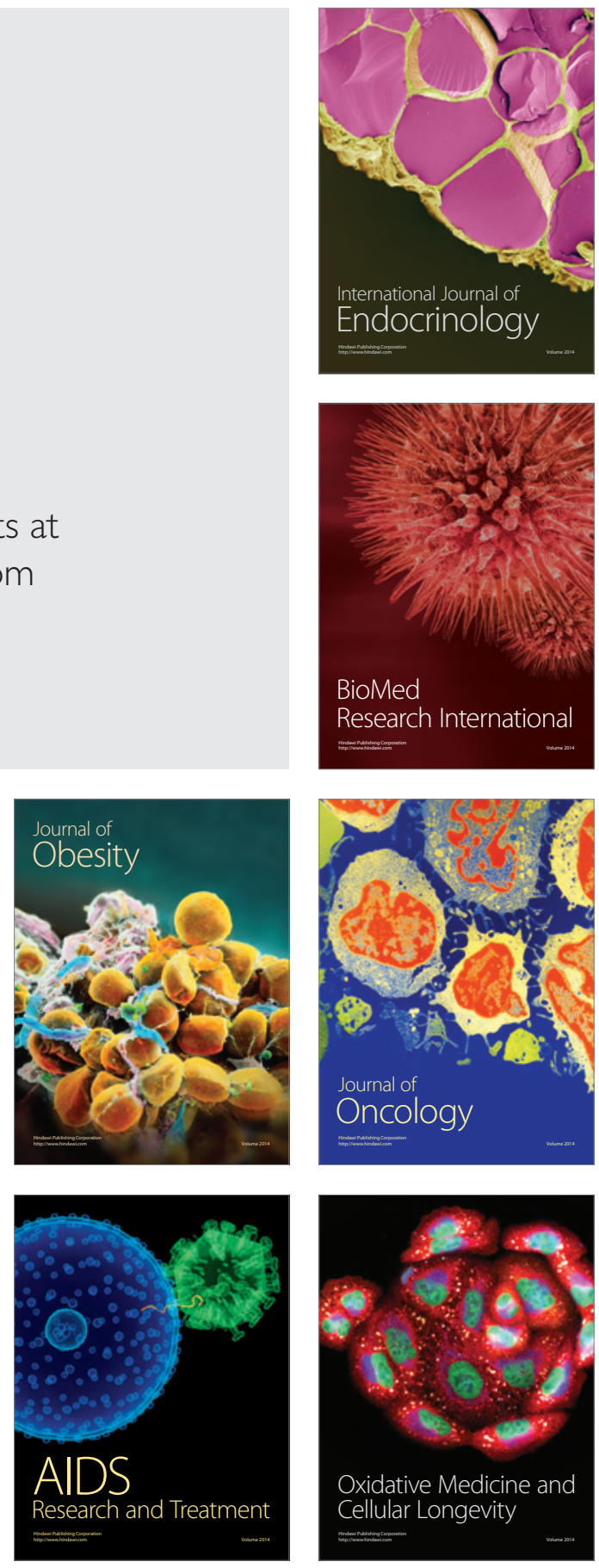